\title{
A Case of Austrian's Triad in the ICU Complicated by Subarachnoid Hemorrhage
}

\author{
Sumit Kapoor ${ }^{\mathrm{a}, \mathrm{d}}$, Adel Mandl ${ }^{\mathrm{b}}$, Jyotsana Thakkarc, Gavin Harris ${ }^{\mathrm{b}}$
}

\begin{abstract}
Austrian triad is a rare syndrome of serious disseminated pneumococcal infection associated with high morbidity and mortality. It is rarely reported in the literature with not many cases. We report a case of Austrian syndrome which got complicated by subarachnoid hemorrhage and death of the patient.
\end{abstract}

Keywords: Endocarditis; Meningitis; Bacteremia

\section{Introduction}

Austrian syndrome, first described by Osler and later described and published in 1957 by Dr. Robert Austrian, is a rare triad of disseminated Streptococcus pneumoniae infection comprised of pneumonia, meningitis and endocarditis [1]. It carries a high mortality rate despite aggressive antibiotic management. We describe the first reported case of Austrian triad admitted to our intensive care unit (ICU) which got complicated by subarachnoid hemorrhage and brain death.

\section{Case Report}

A 55-year-old homeless male with unknown past medical history was admitted to our ICU with altered mental status. He underwent endotracheal intubation for airway protection. CT of head was done on admission showing acute left occipital

Manuscript accepted for publication October 22, 2015

aDepartment of Pulmonary/Critical Care, Baylor College of Medicine, Houston, TX 77030, USA

${ }^{b}$ Department of Internal Medicine, Jacobi Medical Center, Albert Einstein College of Medicine, NY, USA

'Department of Nephrology, North-Shore Long Island Jewish Hospital, NY, USA

${ }^{\mathrm{d} C}$ Corresponding Author: Sumit Kapoor, Department of Pulmonary/Critical Care, Baylor College of Medicine, Houston, TX 77030, USA.

Email: drkapoorsumit@gmail.com stroke. MRI of brain showed multiple areas of stroke in acute ischemia in the left occipital lobe, right anterior midbrain, pons, left side of the medulla, right superior cerebellar hemisphere and left centrum semiovale (Fig. 1-3). Lumbar puncture results were consistent with $S$. pneumoniae meningitis. Further workup revealed pneumococcal bacteremia and pneumonia. Transthoracic echocardiogram showed a mobile 10 $\mathrm{mm}$ vegetation on the mitral valve. His HIV results and urine toxicology screen on admission were negative. He remained hemodynamically stable and received empiric antibiotics and steroids but showed no improvement in mental status. He was evaluated by cardiology and cardiothoracic surgery but no plans for surgical intervention were made since he remained hemodynamically stable. His course got complicated by sudden massive subarachnoid hemorrhage leading to immediate brain herniation and death.

\section{Discussion}

Pneumococcal disease remains a significant cause of morbid-

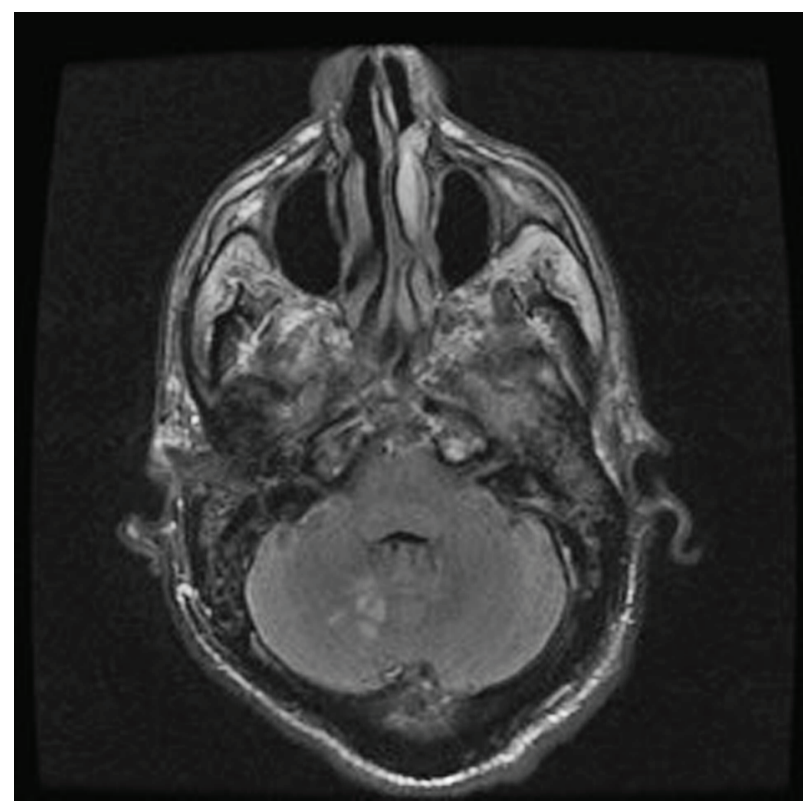

Figure 1. MRI of brain showing acute stroke in left occipital region. 


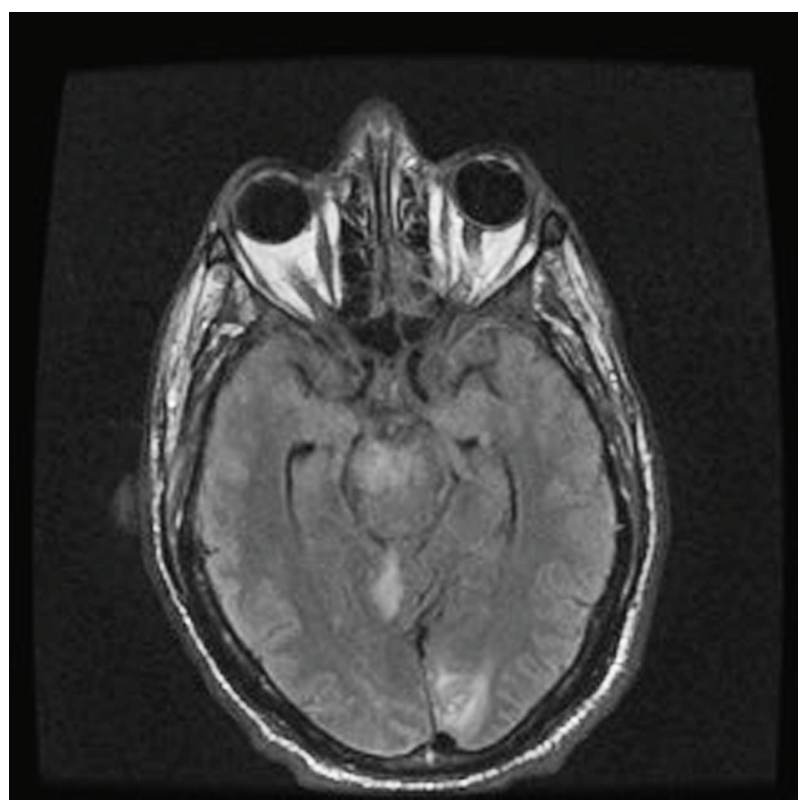

Figure 2. MRI Brain showing acute stroke in midbrain and pons.

ity and mortality. Austrian syndrome is a rare manifestation of invasive disseminated pneumococcal infection comprised of the triad of pneumonia, endocarditis and meningitis. First published in 1957 by Dr. Robert Austrian, it is a rare complication in today's antibiotic era but carries a high mortality around $60 \%$ [1]. Austrian's syndrome remains rarely reported in the literature.

Aronin et al mentioned that 116 or $59 \%$ of the 197 reviewed cases of pneumococcal endocarditis had concurrent meningitis and that 29 of those had Austrian's triad [2]. A review by Gonzalez-Juanatey et al identified two cases of Austrian's syndrome over a 15-year period at their institution in Lugo, Spain and reviewed an additional 16 well-defined cases from the literature [3]. Their review confirmed that alcoholism and liver cirrhosis are significant risk factors for invasive pneumococcal disease, occurring in eight out of 16 patients. The aortic valve was involved in 12 out of 16 patients and valve replacement was required in eight out of 12 patients. Other significant risk factors include sinus infection, immunosuppression, splenectomy and IV drug abuse $[4,5]$.

Patients typically are chronically ill males in their fifth to sixth decade. Complications include heart failure, suppurative pericarditis, tamponade and septic emboli $[6,7]$. Endocarditis is usually left-sided and involves aortic valve more commonly. Embolic events occur in $22-50 \%$ of cases of endocarditis [7]. Given high mortality and complications, early valve replacement should be considered.

Our patient had mitral valve involvement on echocardiogram and did not undergo surgery since he remained hemodynamically stable. His ICU course got complicated by sudden massive subarachnoid hemorrhage, brain herniation and death. Subarachnoid hemorrhage has not been described in the literature as a possible complication of this syndrome. The pathophysiologic mechanism includes either rupture of mycotic aneurysm or septic embolic infarction.

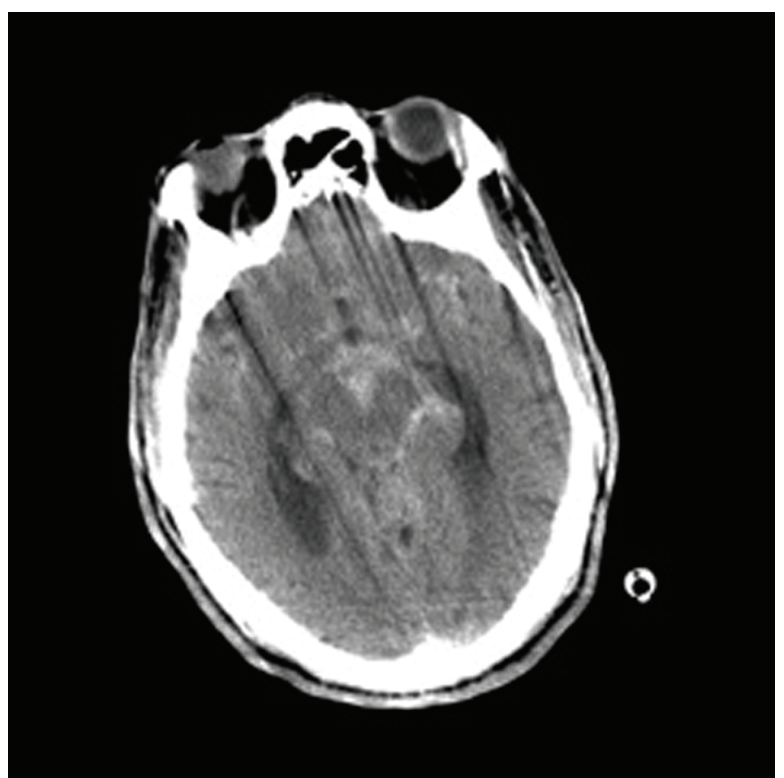

Figure 3. CT of head showing acute subarachnoid hemorrhage.

\section{Conclusion}

Austrian's syndrome should be considered early in patients with S. pneumoniae bacteremia and altered mental status in patients with risk factors. Patients are usually very sick and need ICU admission. It carries a high complication and mortality rate, despite early antibiotics. If surgery is indicated, early valve repair may decrease mortality. Pneumococcal vaccination does provide protection from invasive pneumococcal disease. We report subarachnoid hemorrhage as a rare complication of this syndrome.

\section{References}

1. Austrian R. Pneumococcal endocarditis, meningitis, and rupture of the aortic valve. AMA Arch Intern Med. 1957;99(4):539-544.

2. Aronin SI, Mukherjee SK, West JC, Cooney EL. Review of pneumococcal endocarditis in adults in the penicillin era. Clin Infect Dis. 1998;26(1):165-171.

3. Gonzalez-Juanatey C, Testa A, Mayo J, Gonzalez-Gay MA. Austrian syndrome: report of two new cases and literature review. Int J Cardiol. 2006;108(2):273-275.

4. Munoz P, Sainz J, Rodriguez-Creixems M, Santos J, Alcala L, Bouza E. Austrian syn-drome caused by highly penicillin-resistant Streptococcus pneumoniae. Clin Infect Dis. 1999;29(6):1591-1592.

5. Nog $\mathrm{R}$ et al. Austrian syndrome in an intravenous drug user: A case report. Infect Dis-eases in Clinical Practice. 2010;18(6):406-407.

6. Vindas-Cordero JP, Sands M, Sanchez W. Austrian's triad complicated by suppurative pericarditis and cardiac tamponade: a case report and review of the literature. Int $\mathrm{J}$ Infect Dis. 2009;13(1):e23-25. 
7. Hristea A, Nicolae D, Luka AI, Constantinescu RM, Arama V, Tanasescu R. Invasive pneumococcal infections:
Austrian syndrome. Rom J Intern Med. 2009;47(1):9396. 